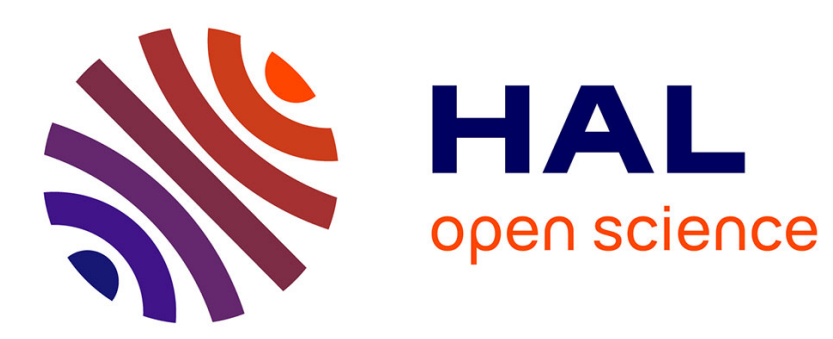

\title{
New insights into Martian dust distribution and water-ice cloud microphysics
}

\author{
Franck Montmessin, Pascal Rannou, Michel Cabane
}

\section{To cite this version:}

Franck Montmessin, Pascal Rannou, Michel Cabane. New insights into Martian dust distribution and water-ice cloud microphysics. Journal of Geophysical Research. Planets, 2002, 107 (E6), pp.5037. 10.1029/2001JE001520 . insu-02897052

\section{HAL Id: insu-02897052 \\ https://hal-insu.archives-ouvertes.fr/insu-02897052}

Submitted on 11 Jul 2020

HAL is a multi-disciplinary open access archive for the deposit and dissemination of scientific research documents, whether they are published or not. The documents may come from teaching and research institutions in France or abroad, or from public or private research centers.
L'archive ouverte pluridisciplinaire HAL, est destinée au dépôt et à la diffusion de documents scientifiques de niveau recherche, publiés ou non, émanant des établissements d'enseignement et de recherche français ou étrangers, des laboratoires publics ou privés. 


\title{
New insights into Martian dust distribution and water-ice cloud microphysics
}

\author{
Franck Montmessin, Pascal Rannou, and Michel Cabane \\ Service d'Aéronomie du CNRS, Paris, France \\ Received 21 May 2001; revised 29 January 2002; accepted 1 February 2002; published 11 June 2002.
}

[1] In this paper we use a direct method which combines microphysics and photometric simulations of the Martian atmosphere. This approach allows us to reproduce vertical profiles of the light scattered by the haze at the limb of Mars. Subsequent results are compared to a multiple-color image provided by the Viking Orbiter camera. The "free parameters" of our study are related to vertical transport and dust size distribution. A sensitivity study has been conducted, encompassing a relevant range of parameters, in order to obtain the most satisfying multispectral modeled profile with respect to the one inferred from data. Except for an unlikely distribution of submicron particles $\left(r_{\text {eff }} \sim 0.2 \mu \mathrm{m}\right)$, no consistent fit can be obtained. This implies that size distributions of dust suggested by previous studies are not in agreement with the present analysis. This apparent discrepancy can be resolved by adding a separate peak of submicron particles to the size distribution previously extracted from Viking Lander images. Only in that case can spectral and vertical structures of haze be successfully matched. While a bimodal distribution would change the current picture of Martian dust, such distribution is a common representation of soil-derived aerosol size function in Earth deserts. We also attempted to derive information from the cloud shown in the Viking limb image. Our estimates suggest that cloud particle effective radius is around $1.2-1.8 \mu \mathrm{m}$, while the cloud visible opacity is 0.02. INDEX TERMS: 0320 Atmospheric Composition and Structure: Cloud physics and chemistry; 0343 Atmospheric Composition and Structure: Planetary atmospheres (5405, 5407, 5409, 5704, 5705, 5707); 5704 Planetology: Fluid Planets: Atmospheres - composition and chemistry; KEYWORDS: Mars, atmosphere, dust, cloud, photometry, microphysics

\section{Introduction}

[2] The study of airborne dust is of prime importance when one's interest centers on Martian climate. Dust particles absorb solar radiations in the visible wavelengths while emitting in the infrared. Thus the thermal structure of the thin Martian atmosphere is significantly affected by the presence of suspended dust particles [Gierasch and Goody, 1972].

[3] A variety of observational techniques has been employed to determine dust optical properties. Significant improvements have been made with regard to the knowledge of the dust seasonal cycle [Fenton et al., 1997; Martin, 1986; Smith et al., 2001]. Few measurements are available to accurately describe vertical structure of dust hazes. Infrared occultation spectrometers during the Phobos mission [Korablev et al., 1993; Chassefière et al., 1995] sounded vertical portions of the atmosphere, providing the first information about the altitude distribution of aerosols. This allowed a quantitative study of the strength of dynamical processes which counteract particle sedimentation. Unfortunately, the short lifetime of the mission meant that it was not possible to obtain a set of data covering a

Copyright 2002 by the American Geophysical Union. 0148-0227/02/2001JE001520 significant period of time and a broad latitudinal range. Jaquin et al. [1986] reduced the Viking Orbiter images of the Martian limb into scattered light versus height profiles. This observation yielded the greatest number of observations concerning the vertical structure of haze. Dusty haze was tracked during more than one Martian year and at practically all latitudes. These data showed the orbital dependence of haze vertical extent. On the other hand, the vertical portion useful for data inversion does not include the lower atmosphere, where hazes are optically saturated. Thus only upper portions of scattered light profiles were used to derive the extinction coefficient of aerosols [Jaquin et al., 1986; Kahn, 1990].

[4] In addition, as condensate clouds were observed in the Martian atmosphere, a number of microphysical models were developed to assess the physical mechanisms governing Martian aerosols [Michelangeli et al., 1993; Colaprete et al., 1999]. Such models highlighted the interactions existing between dust and water-ice particles. These models were successful in simulating the formation of water-ice clouds, showing several features similar to observations. Good values were obtained for the average size of ice particles, cloud opacity, and water vapor profile [Rodin et al., 1997; Pearl et al., 2001]. Furthermore, while investigating cloud radiative effects, Colaprete and Toon [2000] were able to explain the temperature inversions observed by 
Mars Pathfinder and Mars Global Surveyor. On Earth, such models were also useful to simulate Polar Stratospheric Clouds [Toon et al., 1989a]. In the present paper we extend the field of comparison between these models and the data. To do so, we have developed a microphysical code dedicated to the Martian atmosphere. This code was briefly described by Bertaux and Montmessin [2001]. Concurrently, we have also modified the photometric model of Rannou et al. [1997]. The combination of the two codes offers a direct method that allows us to simulate limb images like those obtained by Viking Orbiter and reported by Jaquin et al. [1986]. Such an approach is used for the first time in the case of Martian aerosols. This study is motivated by the will to highlight the constraints supplied by microphysics when deriving aerosol characteristics. The first section of this work introduces the theoretical developments that underlie microphysical mechanisms. Section 2 presents a brief description of the models employed for this study. In section 3 we present results of microphysics/ photometry simulations. The purpose is to determine the best size distribution of dust which gives a modeled haze profile matching data. We discuss the relevance of the widely accepted size distributions for dust. Since a condensate cloud can be identified in the multiple-color image of Jaquin et al. [1986], estimations of cloud particle size as well as visible opacity are given by our analysis.

\section{Microphysics}

[5] Martian water-ice clouds have significant similarities to terrestrial cirrus clouds. They both form onto foreign substrates, assuming that Martian dust particles are the cloud condensation nuclei [Gooding, 1986]. The formation of Martian clouds is the result of many microphysical processes, including heterogeneous nucleation, condensation and sublimation, gravitational settling, and dynamics.

\subsection{Nucleation Theory}

[6] In the following, one defines the saturation ratio $S$, at temperature $T$, as the ratio $p / p_{\text {sat }}$ between $p$, the actual pressure of water vapor and the saturation pressure $p_{\text {sat }}$ at temperature $T$. The transformation of a vapor species into its liquid or solid phase does not occur the instant the free energy of the condensed phase is lower than that of vapor. This transformation first consists in the clustering of vapor monomers, that is, the formation of small nuclei assumed to be spherically shaped. The associated free energy change $\Delta F$ in the case of transformation into ice depends on the cluster radius $r$ as well as on the surface tension $\sigma_{\mathrm{vi}}$ between ice and vapor $(0.12 \mathrm{Nm}$ for water ice). Phase change occurs when $\Delta F$ is at its minimum $\Delta F^{*}$, that is, when nucleus radius exceeds a critical equilibrium value $r^{*}$, enabling spontaneous growth by condensation. The onset of nucleation is described by the rate per unit of time $J$ at which embryos cross the critical size.

[7] If clusters form on the surface of an insoluble substrate, they enlarge their curvature radius and consequently nucleate more readily than on their own, namely, homogeneously. The contact between an insoluble support and an icy embryo is approximately described by an angle $\theta$ yielding the free energy factor $m(=\cos \theta)$. The correspond- ing $\Delta F^{*}$ is lowered by a factor $f$ (see Pruppacher and Klett [1978] for details) which dramatically depends on $m$ and on the ratio of curvature radii of the cluster onto that of the particle. In most cases the presence of dust in the Martian atmosphere promotes but restricts nucleation to occur heterogeneously.

[8] Assuming that water molecules join an embryo during their motion upon the substrate, the nucleation rate is expressed as follows [Keese, 1989]:

$J_{s d}=Z \frac{n_{\mathrm{H}_{2} \mathrm{O}}^{2} k T}{\dot{m}_{w} \nu_{s} f} S_{p} r * \bar{\delta} \sin \theta \cdot \exp \left[\frac{-\left(\Delta F^{*}+\Delta F_{\text {sd }}-2 \Delta F_{\mathrm{des}}\right)}{k T}\right]$,

where $n_{\mathrm{H}_{2} \mathrm{O}}$ is the number density of water in the atmosphere, $k$ is the Boltzman constant, $Z$ is the Zeldovitch factor accounting for the steady state nature of nucleation process, $\dot{m}_{w}$ is the mass of a water molecule, $\nu_{s}$ its jump frequency $\left(\sim 10^{13} \mathrm{~s}^{-1}\right)$ onto the substrate, $S_{p}$ is the surface area of the substrate, $\bar{\delta}$ is the average distance traveled by a molecule in a diffusion step or jump, $\Delta F_{\text {des }}$ is the molecular desorption energy $\left(\sim 6 \times 10^{-20} \mathrm{~J}\right.$ [Mooney et al., 1952; Anderson et al., 1978; Zent and Quinn, 1997]), and $\Delta F_{s d}$ is the molecular energy of surface diffusion $\left(\sim \Delta F_{\mathrm{des}} / 10\right.$ [Seki and Hasegawa, 1983]). This expression is used in our model.

[9] Because of the bottleneck statute of nucleation in phase transformation, microphysical computations are highly sensitive to the uncertainties on parameters such as $m$ [Michelangeli et al., 1993]. These authors ranged m within 0.925-0.975 as did Colaprete et al. [1999]. Our reference $m$ value follows these studies, namely, 0.945 , although no published data can provide an experimental approval. Moreover, the classical nucleation theory based on the approximation of spherical particles neglects surface nonhomogeneities, omitting that concavities are the preferred nucleation sites (inverse Kelvin law). It is also limited by its macroscopic point of view since surface tension and contact angle are not adequate physical quantities to characterize embryos made of few molecules. Heterogeneous nucleation might also be completed by ion-induced and homogeneous nucleation. Except for extremely low temperatures such as those of the polar night, homogeneous nucleation is not likely to occur in the Martian atmosphere (Figure 1). Also, despite a brutal drop-off of ion concentration below $50 \mathrm{~km}$ [Zhang et al., 1990], ion-induced nucleation should be envisioned as a possible option for forming ice crystals. The lack of ionic measurements in the lower layers of the Martian atmosphere precludes a precise assessment of this phenomenon.

\subsection{Condensation Theory}

[10] Nucleation, the first step of the phase transformation, is followed by condensation. The classical description of spontaneous growth through the condensation process mainly focuses on the case of vapor turned into liquid. This classical description, though not matching all the physical processes at work when vapor turns into ice, isused in our model. Thus either condensation or evaporation implies a radius variation of the water ice crystal, the initial size of which is assumed to be that of the support it has nucleated on. This radius change is given by the following expression 


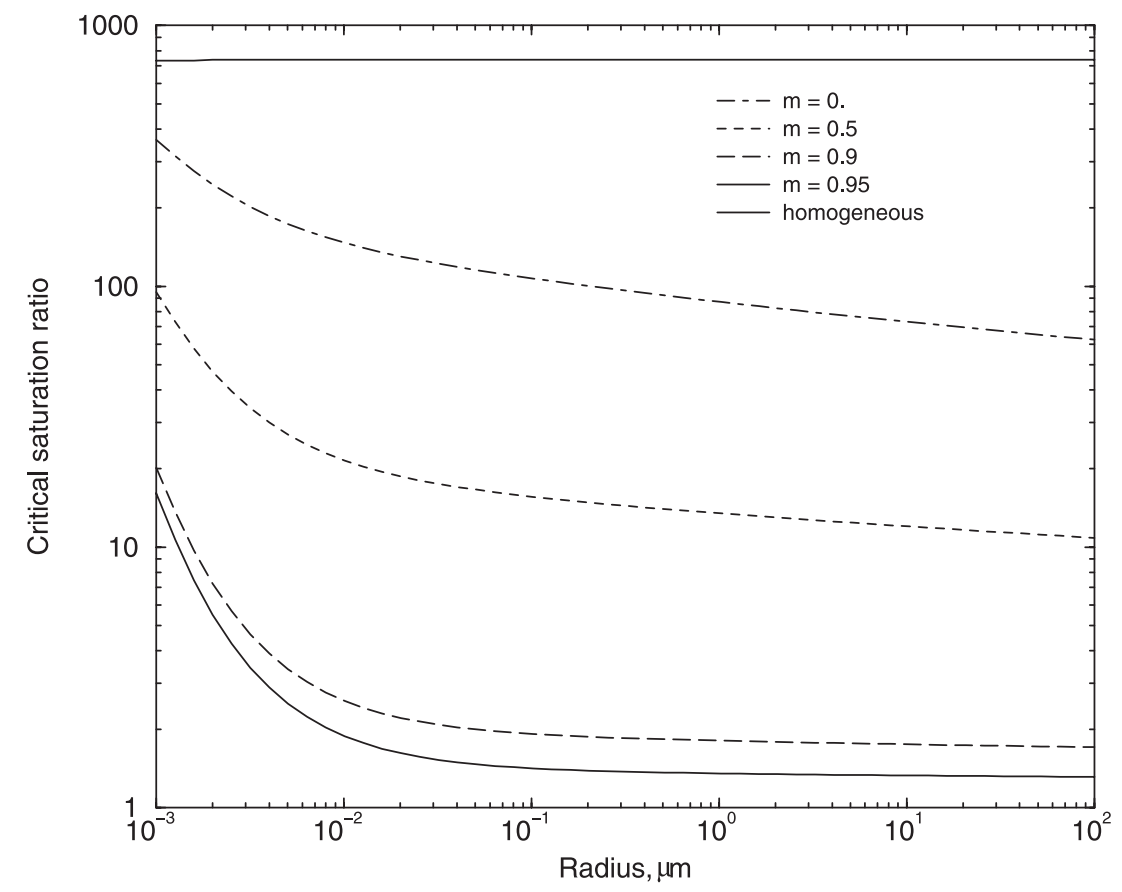

Figure 1. Nucleation curves as a function of condensation nucleus size for different contact parameter (m) values. Critical saturation ratio is given by 1 embryo $\mathrm{cm}^{-2} \mathrm{~s}^{-1}$ (heterogeneous case) and 1 embryo $\mathrm{cm}^{-3} \mathrm{~s}^{-1}$ (homogeneous case). The homogeneous curve is the asymptotical limit of the heterogeneous nucleation expression. If condensation nuclei number is large enough, water vapor nucleation on its own is not expected to occur.

derived from Pruppacher and Klett [1978] and MacKenzie and Haynes [1992]:

$$
r \frac{d r}{d t}=\frac{S-S_{e q}}{F_{d}+F_{h}+F_{k}},
$$

where

$$
F_{d}=\frac{\rho_{i} k T_{\infty}}{D^{\prime} \dot{m}_{w} p_{s a t}\left(T_{\infty}\right)}
$$

represents the molecular diffusion contribution term, where $\rho_{i}$ is the water-ice density $\left(917 \mathrm{~kg} \mathrm{~m}^{-3}\right)$ and where $D^{\prime}$ is the corrected molecular diffusivity which accounts for the possibility of a molecule diffusing through a continuum or a kinetic medium,

$$
F_{h}=\frac{L_{i} \rho_{i}}{K T_{\infty}}\left(\frac{L_{i} \dot{m}_{w}}{k T_{\infty}}-1\right)
$$

is the heat diffusion contribution term, where $K$ is the thermal conductivity of air, $S_{\text {eq }}$ is

$$
S_{\mathrm{eq}}=\exp \left(\frac{2 \sigma_{v i} \dot{m}_{w}}{k T_{\infty} \rho_{i} r}\right)
$$

where $T_{\infty}$ is the temperature far in distance from the ice crystal. $F_{k}$ is the surface kinetics contribution term, assumed to be zero for computations.

[11] In the future developments of the Martian microphysics model, a significant improvement will certainly be accomplished when accounting for surface kinetics (through the $F_{k}$ term). The surface kinetics effect is a result of the specific growth mechanism of ice crystals. Indeed, water molecules within the condensing flux are not instantaneously integrated to the ice bulk as they are during liquid transformation. After deposition, water molecules must diffuse across the crystal surface in order to join a site where they are strongly bound if not being desorbed during their travel. The theoretical developments of Kuroda [1984] show that this effect could be prominent in the determination of the growth rate. The lack of experimental support precludes any modification of the classical expression of growth rate. Also, it also turns out that a more accurate assessment of surface kinetics would be needed to definitively adopt the most appropriate water-ice condensation scheme in microphysical models.

\subsection{Vertical Transport and Particle Aggregation}

[12] The settling velocity for ice and dust particles, $\omega$, is given by the Stokes relationship corrected by the Cunningham slip-flow factor. Fuchs [1964] gives

$$
\omega=\frac{2 g \rho_{p}}{9 v_{a}}\left\{1+K n\left[1.246+0.42 \exp \left(\frac{-0.87}{K n}\right)\right]\right\} r_{p}^{2},
$$

where $\omega$ is the settling velocity, $K n$ is the Knudsen number, $g$ is gravity, $\rho_{p}$ is the particle density, $r_{p}$ is the radius of the particle, and $\nu_{a}$ is the dynamic viscosity of air. Velocity increases with particle radius and altitude. Obviously, mean particle radii at cloud levels result from the combination of the condensation growth and the vertical transport char- 
acteristic times. Since sedimentation brings ice particles to lower altitudes where sublimation might occur, this process is responsible for the water vapor enrichment in these regions.

[13] Atmospheric dynamics implies an additional transport embracing several phenomena like turbulent mixing or convection. Hence we use eddy mixing to represent dynamics within the atmospheric column while parametrizing it by the coefficient $k_{d}$.

[14] Another microphysical process of interest is particle coagulation. Both ice and dust particles may collide either by Brownian motion or by settling velocity difference. On Earth this latter process allows water droplets to reach greater particle sizes (from 10 to $100 \mu \mathrm{m}$ ) than would condensational growth alone. Michelangeli et al. [1993] have shown that coagulation involves only particle radii lower than $0.1 \mu \mathrm{m}$. Thus coagulation will not be considered significant in the cloud formation or evolution. However, this point is discussed more extensively in the next paragraph.

\subsection{Dust Distribution}

[15] In this study the generic term "dust model" is defined by the average size distribution of dust aerosols in an atmospheric column. A dust model is represented by the first two moments of the corresponding size distribution, namely, the geometric cross-section weighted mean particle radius (the effective radius $r_{e f f}$ ) and the effective width of the distribution $\left(v_{\text {eff }}\right)$. Previous studies chose various types of functions to derive size distributions. Toon et al. [1977] and Clancy et al. [1995] employed gamma-modified functions, while Pollack et al. [1995] chose the lognormal form to extract a dust model. However, Hansen and Travis [1974] have shown that different forms of functions having the same first two moments $\left(\mathrm{r}_{\text {eff }}\right.$ and $\nu_{\text {eff }}$ ) lead to the same single scattering properties. Thus these authors define a size function derived from the gamma-modified formulation, where the effective radius and width are the only parameters. Recently, Tomasko et al. [1999] and Markiewicz et al. [1999] adopted this function. Whereas this size function is particularly convenient for optical data retrievals, its use is not appropriate for microphysical simulations. As stated by Tomasko et al. [1999], this simplified gamma function leads to an unphysically large amount of small particles for effective widths larger than 1/3. Furthermore, it can be demonstrated that different forms of functions whose first two moments are identical do not induce the same altitude distribution of aerosols.

[16] With regard to the problem of choosing a consistent form of function, it is not possible to specify size distribution parameters without accounting for some elementary physical constraints. As explained previously, dust particles larger than $0.1 \mu \mathrm{m}$ are essentially not affected by the coagulation process. The dust model advocated by Clancy et al. [1995] is highly unstable from this point of view. Figure 2 reports the temporal evolution of this size distribution experiencing the only effect of coagulation under Martian-like conditions. After 10 days of simulation the smallest particles have collided with larger ones. The function almost loses its initial asymmetrical shape as it tends naturally toward a lognormal form. This result is in

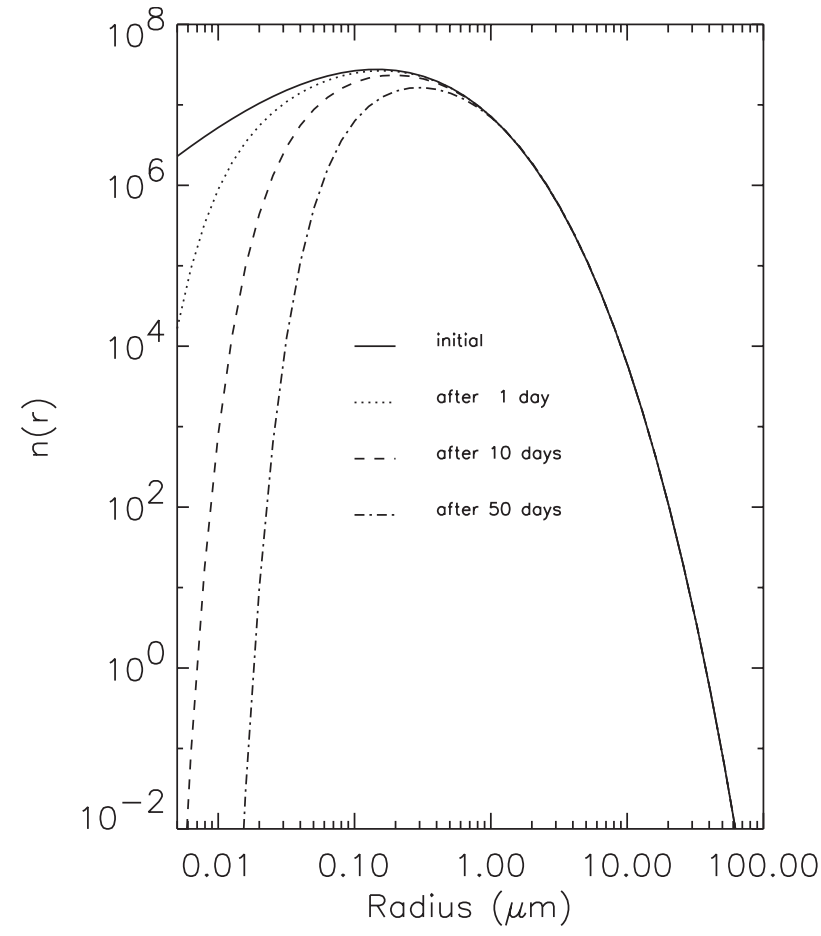

Figure 2. Modeled evolution of the gamma-modified size function of dust particles reported by Clancy et al. [1995] $\left(\mathrm{r}_{\text {eff }}=1.8 \mu \mathrm{m}, \nu_{\text {eff }}=0.8\right)$ under the only influence of Brownian coagulation.

agreement with Friedlander [1977]. This author found that the "self-preserving" size distribution, that is, the asymptotic form approached after long times, of particles under the process of Brownian coagulation is approximately lognormal. Furthermore, a lognormal function is also the asymptotic solution of particle size distribution for the breakage mechanism Epstein [1947], which is a basis process for sandblasting. Because of these considerations, we assumed a lognormal distribution for Martian dust throughout this study.

\section{Description of Models}

\subsection{Microphysics Model}

[17] The microphysical model is derived from the Titan aerosol formation model engineered by Cabane et al. [1992] and is based on that of Toon et al. [1988]. The atmospheric column is divided into 32 vertical layers of equal thickness $(2 \mathrm{~km})$, except near the ground, where the grid is further refined. Thermal profiles and their related diurnal variations, which are the most constraining inputs for water-ice cloud formation, are provided by the Viking scenario of the European Martian Climate Database (EMCD) [Lewis et al., 1999]. The particle radius grid (dust particles and ice crystals) is based on 50 size bins, using a volume ratio of 2 between two adjacent bins. All microphysical processes described in the previous section, except coagulation, are represented in the model. Iterative computations are then performed until a steady state is obtained. In this way, number densities of aerosols are obtained for all particle size bins and for all atmospheric 
layers. These quantities are stored in a two-dimensional (2-D) array dedicated to a given type of aerosol (dust or water ice). A specific array was also included to account for the presence of a dust core within ice crystals.

[18] The model does not allow particles to reach the ground, keeping them confined in the lowest layer of the model. These particles are then redistributed by eddy mixing. This could be interpreted as a rough representation of particle lifting as suggested by Colaprete et al. [1999].

\subsection{Photometry Model}

[19] Vertical resolutions of aerosols have already been retrieved from observations at the Martian limb [Anderson and Leovy, 1978; Jaquin et al., 1986], providing some information about hazes and clouds. On the other hand, a direct step based on the combination of a 1-D microphysical model along with a radiative transfer model allows one to simulate the observation of a limb haze. Such a method offers strong constraints since modeled haze structures explicitly include microphysics constraints.

[20] Rannou et al. [1997] have synthetically reproduced several limb observations of Titan hazes. Using a code of microphysics [Cabane et al., 1992] to simulate haze structure and optical properties, they deduced intensity profiles by adding a light-scattering model at the limb inspired by the work of Rages and Pollack [1983]. For a detailed description of the photometric code the reader is referred to Rannou et al. [1997].

[21] In our study we follow an identical procedure to reproduce one of the limb profiles reported by Jaquin et al. [1986] (henceforth J86). Synthetic profiles result from the use of our microphysical code combined with the photometric code presented below.

[22] Any incident solar ray traveling through atmospheric slabs toward the ground experiences extinction mostly due to scattering (in the visible) by particles. The portion of flux which is scattered with an angle $\theta$ toward the observer along its line of sight encounters additional extinction when it emerges from the atmosphere. Consequently, a measured flux at a given altitude integrates light intensity originating from each of all the slabs that the slant path goes through. Reproducing an emergent flux as a function of elevation requires many physical quantities of the particles distributed among the sounded portion of the atmosphere. These quantities are the optical depth $\tau_{0}$ and $\tau_{1}$ along the incident and emergent paths, respectively, both deduced from the extinction coefficient $\beta$ computed for each slab. Also needed is the value of $\bar{\omega} P(\theta)$, the product of the single scattering albedo $\bar{\omega}$ by the phase function $P$ at the scattering angle $\theta$.

[23] Once microphysics computations are carried out, subsequent aerosol distributions are reduced in order to obtain the optical properties of interest. Mie theory is used for water-ice particles and for dust particles having a size parameter $x(x=2 \pi r / \lambda)$ lower than 5 , as suggested by Tomasko et al. [1999]. Following these authors the scattering cross sections of larger dust particles were enhanced by a factor of 1.3 to account for the assumed nonspherical shape of dust within this size range. Refractive indices were taken as used by Warren [1984] for ice and as used by Ockert-Bell et al. [1997] for dust.
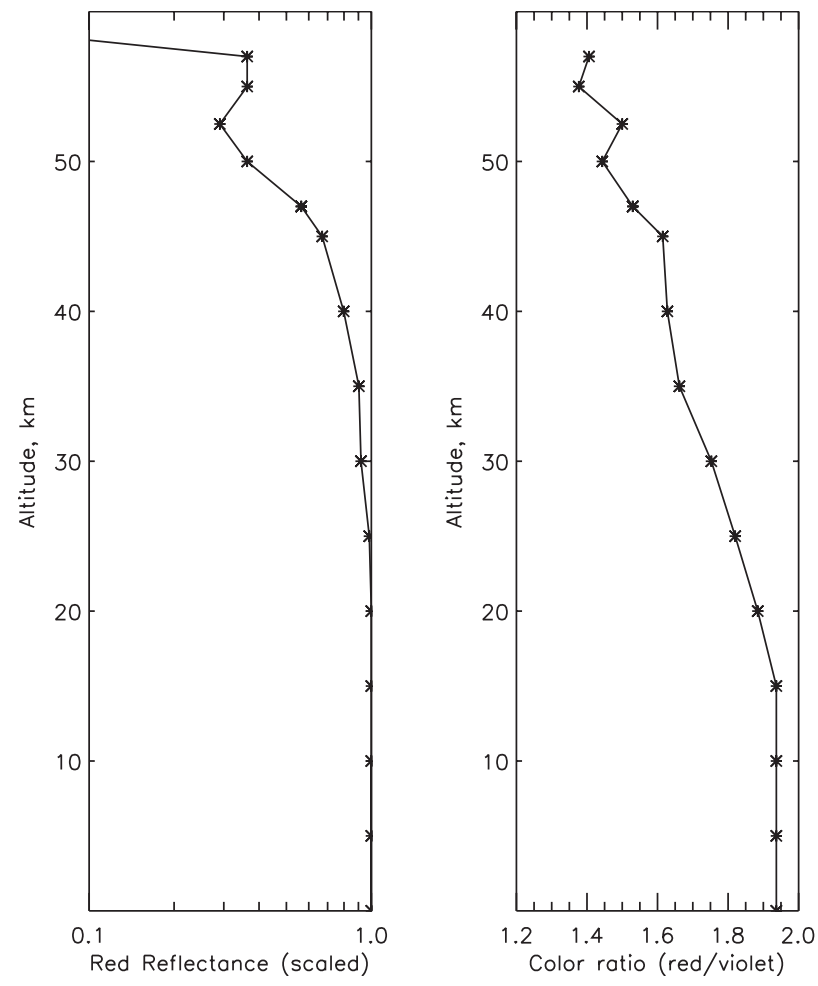

Figure 3. Limb data taken from Jaquin et al. [1986, Figure 6]. The left panel shows the scattered light intensity profile for the red channel (scaled by its value near the ground). The right panel shows the ratio of red onto violet reflectance as a function of height. Observations were made in the southern hemisphere $\left(\sim 15^{\circ} \mathrm{S}\right)$ during winter $\left(L_{s}=\right.$ $176^{\circ}$ ). The detached layer above $50 \mathrm{~km}$ is probably the cause of a water-ice cloud.

[24] Finally, $I / F(z)$ is computed at the $n$th line of sight by combining the contributions mentioned above, that is,

$$
\begin{gathered}
I / F_{n}=\sum_{i=1}^{2 n} \int_{x_{i}-1}^{x_{i}} 0.25(\bar{\omega} P(\theta))_{j} \exp \left(-\tau_{0 i}-\tau_{1 i}\right) \beta_{j} d x, \\
j=i \text { if } i<n+1(j \text { from } 1 \text { to } n), \\
j=2 n+1-i \text { if } i>n(j \text { from } n \text { to } 1) .
\end{gathered}
$$

[25] Multiple scattering is also taken into account as a factor in enhancing intensity in each slab by using the twostream approximation of Toon et al. [1989b].

\section{Observational Data and Results}

\subsection{Data Description}

[26] The radiometric calibration of Viking cameras provided reflectance accuracies to $7 \%$ absolute, allowing meaningful data analysis (J86). Only one multiple-color image of the limb was reported by J86 (their Figure 6, our Figure 3). This figure illustrates a haze extending up to an altitude of $60 \mathrm{~km}$, where the presence of a detached layer is well pronounced. J86 supposed this detached layer to be the result of a condensate cloud. This observation was per- 
formed in the southern hemisphere $\left(15^{\circ} \mathrm{S}\right)$ before the first planet-encircling dust storm $\left(L_{s}=176^{\circ}\right)$. Simultaneous soundings of the same limb at several wavelengths make it possible to obtain a more accurate estimation of the type of aerosol from which the probed haze is composed. Using the color ratio, one is able to go back to the size of the particle assumed to scatter light at a given elevation as explained later in this section. To do so, one must account for the scattering properties of aerosols (see section 3.2 for details). This method is less straightforward than that used for solar occultation data retrieval. The latter is indeed much less sensitive to the shape of the considered particle since the extinction efficiency $Q_{\text {ext }}$ is the only property of interest [Korablev et al., 1993]. This explains why the limb data of J86 did not give the same amount of information about Martian dust distribution as the Phobos data did.

[27] As raised by Forget et al. [1999], the calculation of atmospheric heating rates for Mars is highly sensitive to even small uncertainties in the radiative properties of airborne dust. The work carried out here adds to the ongoing efforts focusing on the determination of the single scattering properties of airborne dust. In the remainder of this study, we concentrate our discussion on the multiple-color image of J86 (Figure 3).

[28] It is instructive to consider the basic morphology of a scattered light versus height profile like the one displayed in Figure 3. Near the top of the reflectance profile, tangential optical depth is small enough to allow direct inversion of the extinction coefficient $\beta(z)$ (where $z$ is the altitude of sounding). By extension, a profile morphology approximately follows the associated vertical distribution of particle concentration, provided that both particle size $r_{\text {eff }}$ and the product $Q_{\text {ext }} \omega P(\theta)$ do not vary significantly with height. According to the strength of the mechanism counteracting sedimentation, particles are more or less present higher in the atmosphere, restricting the extent of the scattered light profile. As outlined in section 3.1, any flux measured at a given height $z$ is the result of light scattered by the aerosols encountered along the line of sight. When the tangential optical depth exceeds some critical value $(\sim 4)$, the amount of light scattered by the deepest region of the slant path has been largely extinguished along its emergent path, therefore not contributing to the flux received by the Orbiter camera. A tangential optical depth of about 4 corresponds approximately to a normal optical depth of 0.1 . Thus a scattered light profile might feature a constant flux portion (referred to as the asymptotic portion) in its lower part, where dust is more confined. It turns out that changes in the width of the asymptotic portion of a profile provide an efficient gauge of dust-loading variations with time. This property is particularly useful for monitoring the onset and the decay of dust storms, as reported by J86. Concerning the multiple-color profile considered in this study, we estimate the normal optical depth of dust to be around unity (consistent with the data of Colburn et al. [1989] at the same period over the Viking Lander site).

[29] Multispectral measurements are necessary to derive single scattering properties of particles forming a haze. The incoming solar flux over a particle is indeed scattered in a way depending on the spectral behavior of the quantity $Q_{\text {ext }} \omega P(\theta)$. For a given phase angle $\theta$, the red to violet ratio of $Q_{\text {ext }} \omega P(\theta)$ is simply a function of the
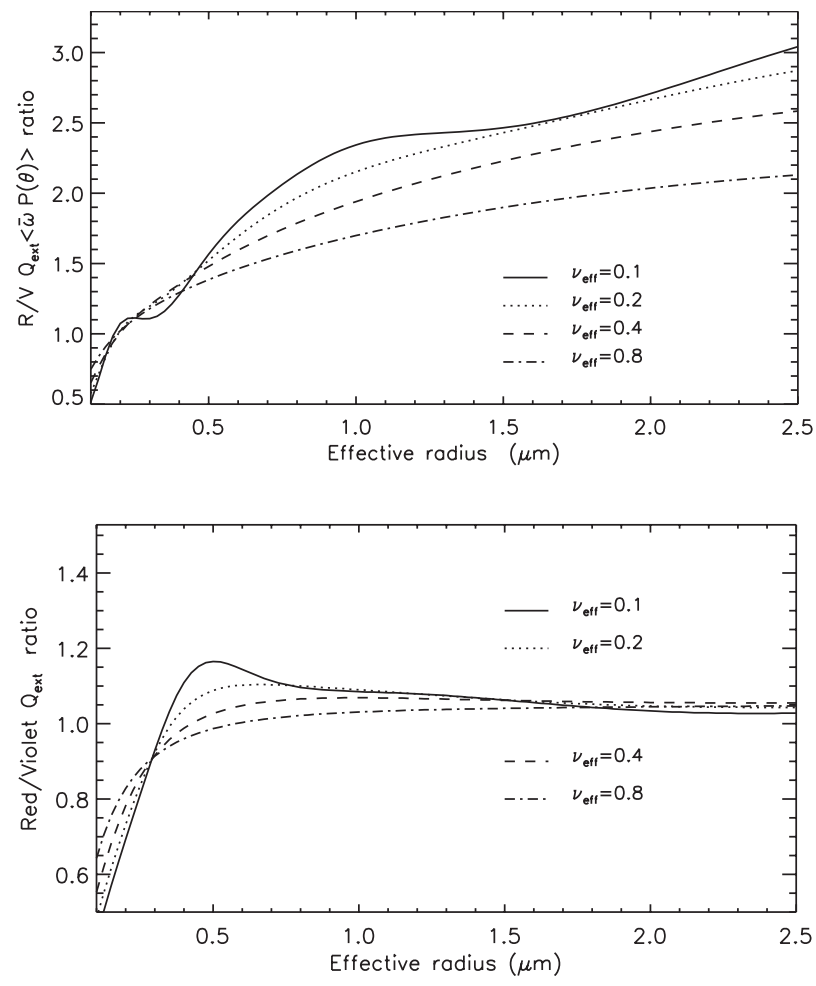

Figure 4. Single scattering properties of dust for a variety of effective radii and variances. The red to violet ratio of the product $Q_{\text {ext }} \bar{\omega} P(\theta)$ (where $\theta$, the phase angle, is taken at $100^{\circ}$ ) as a function of $r_{\text {eff }}$ is shown in the upper graph. The sensitivity to several values of $\nu_{\text {eff }}$ is shown by different curves, as labeled. The red to violet ratio of the efficiency parameter $Q_{\text {ext }}$ is shown in the lower graph.

particle size parameters (see upper graph of Figure 4). The principal characteristic of this ratio is to increase with increasing particle size. In keeping with that fact, particle size could be straightforwardly inferred from the observed color ratio at the elevation of measurement. On the other hand, as this incoming flux penetrates through layers of particles, it possibly suffers contrasted extinction with regards to its spectral pattern. The extinction efficiency $Q_{\text {ext }}$ of particles depends essentially on the value of $x=2 \pi r / \lambda$. For large values of $x$, that is, the limit of geometrical optics, $Q_{\text {ext }}$ is almost constant. There is a range of $x$ values for which $Q_{\text {ext }}$ increases rapidly (Rayleigh extinction) with increasing radius or/and decreasing wavelength $\lambda$, as illustrated by the bottom graph of Figure 4. Theoretically, particles smaller than $0.4 \mu \mathrm{m}$ extinguish preferentially bluer wavelengths, whereas larger particles are almost neutral within the range of visible wavelengths.

[30] Inspection of the J86 data indicates a constant color ratio of $\sim 1.9$ between the ground and $15 \mathrm{~km}$ and a decrease in ratio toward a value of 1.4 at $50 \mathrm{~km}$. As water ice is essentially nonabsorbing in the wavelength range of the investigations, its color ratio is expected to be lower than dust for an identical particle size (compare Figure 5 to Figure 4). This property is balanced by the fact that condensed water adds mass to the dust nuclei which it 

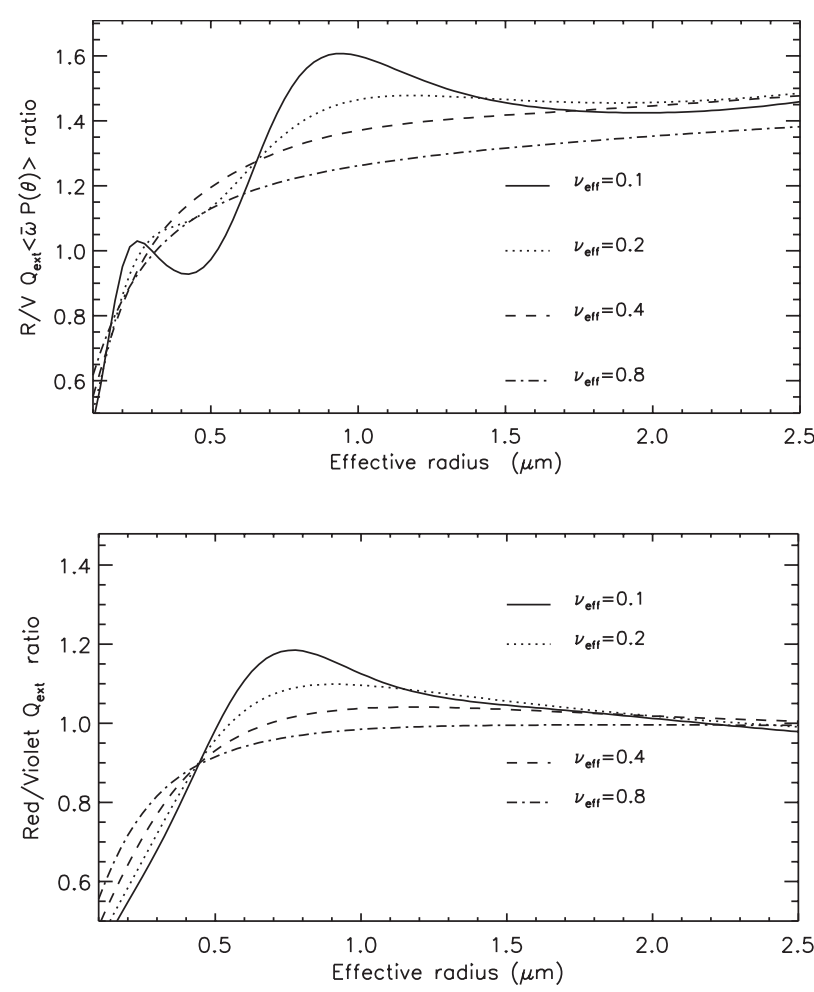

Figure 5. Same as Figure 4 but for the single scattering properties of ice.

nucleates onto, therefore enlarging the mean size and the corresponding color ratio of local particles. A significant variation of color ratio is thus not a systematic signature of condensate cloud. One must recall that color ratio variation with height can also be caused by vertical variations of dust particle effective size. Figure 3 shows that color ratio increases somewhat in the vicinity of the detached layer $(z \sim 50 \mathrm{~km})$ but not at a noticeably larger rate than the observed decrease in the continuous portion of the profile (between 15 and $50 \mathrm{~km}$ ). Thus the basic assumption that water-ice clouds are bluer than the underlying dust haze might be too rough to describe properly the vertical structure of Martian hazes. Dust particle size variation with height contributes just as much to the vertical behavior of color ratio. The latter is not systematically indicative of changes in particle composition. For instance, a deeper inspection of Figure 3 reveals a bump in the color ratio profile above $35 \mathrm{~km}$. A similar trend is observed though less discernable in the intensity profile. There is no clear interpretation for such a rupture in the color ratio structure. Several causes could provide potential explanations: horizontal transport contributions, condensate particles implying the presence of a secondary water-ice cloud, and dynamical effects such as updraft winds creating departures from the more gradual morphology of mixed hazes.

[31] The principal aim of our work consists in deriving parameters of a dust model able to fit the observation of J86. The classical task of intensity data inversion is rarely constrained by physical mechanisms such as vertical redistribution of particles or by the interactions between different kinds of particles (nucleation of ice upon dust cores, dust removal through ice particle sedimentation). Hence, when we test the size parameters ( $\mathrm{r}_{\text {eff }}$ and $\left.\nu_{\text {eff }}\right)$ of a dust model, the corresponding modeled haze structure we use for comparison with data explicitly accounts for microphysical constraints. By adding cloud simulation, we allow further comparisons of the corresponding modeled cloud to the detached layer shown in the data (Figure 3). To do so, we explore a space of effective radii, bounded by a lower value of $0.1 \mu \mathrm{m}$ and a higher value of $2.5 \mu \mathrm{m}$. In the same way, we allow effective width to vary between 0.03 and 1 .

[32] Owing to the very low timescales of nucleation or condensation, cloud simulation is highly computer-timeconsuming. Hence a consistent search within the parameter space we adopted is not suitable to simulations enabling the whole microphysics package. Thus we first restricted our analysis to the color behavior and the morphology of the continuous portion of the haze of J86, that is, the region between the ground and $50 \mathrm{~km}$, while neglecting nucleation/ condensation processes.

\subsection{Simulations Without Water-Ice Cloud}

[33] A preliminary estimation of the best fitting dust model is performed using a simplified microphysical scheme where cloud formation is disabled. Assuming no updraft wind, we conducted simulations within the context of three mixing scenarios displayed in Figure 6. The standard one employs a constant mixing coefficient $\left(100 \mathrm{~m}^{2} \mathrm{~s}^{-1}\right)$ between the ground and $25 \mathrm{~km}$ which increases inversely with density toward the top of the atmosphere, where its value is $\sim 6000 \mathrm{~m}^{2} \mathrm{~s}^{-1}$ at $60 \mathrm{~km}$. An intermediate scenario

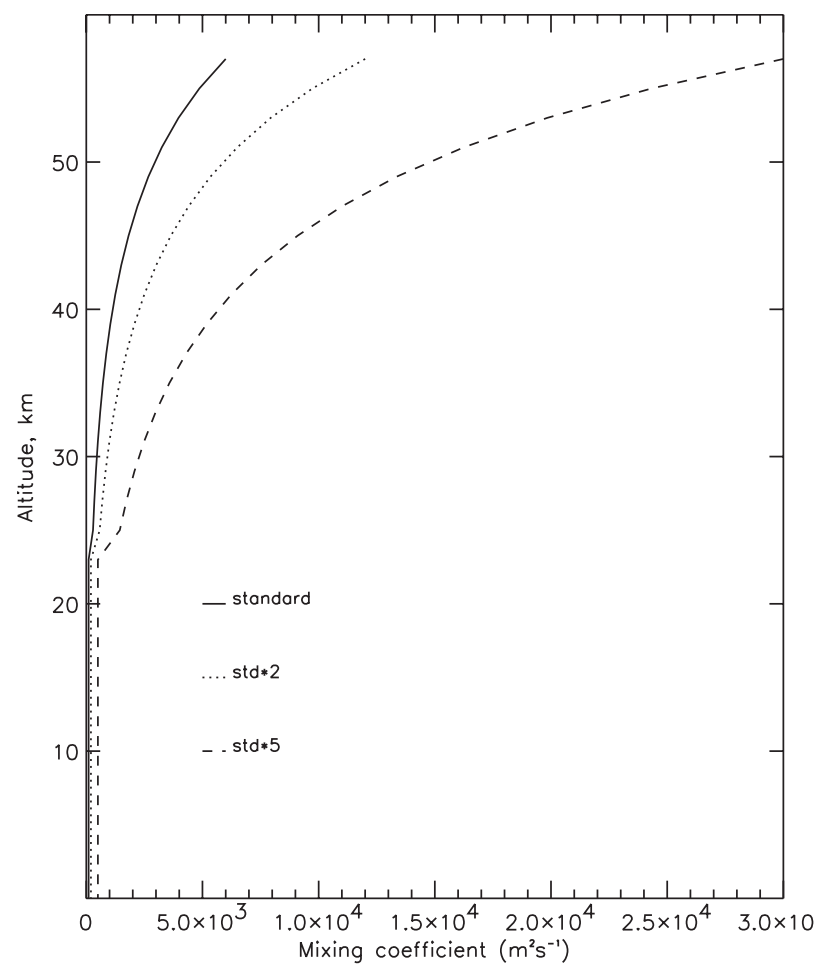

Figure 6. A summary of the three mixing scenarios used in this work, differing by their strength. Eddy-mixing coefficients are plotted versus height. 


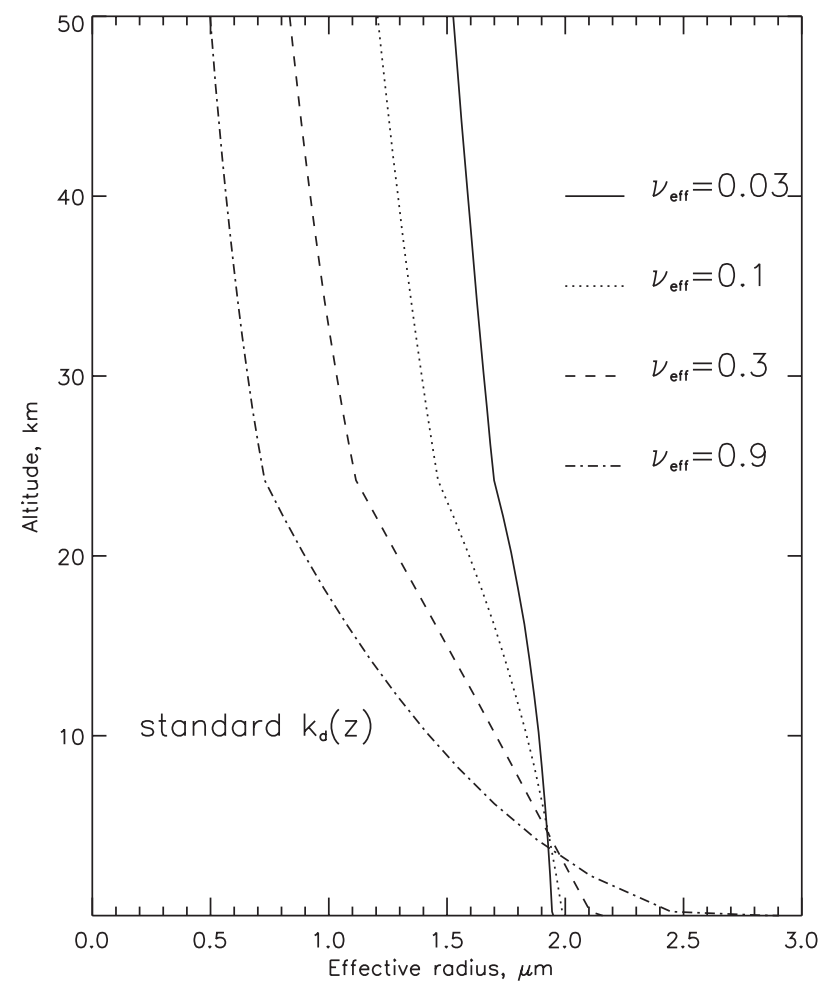

Figure 7. The effect of varying the effective variance $\nu_{\text {eff }}$ for a dust model. At the inital state (not shown), all dust models have the same effective radius $\left(r_{\text {eff }}=1.8 \mu \mathrm{m}\right)$ and a given $\nu_{\text {eff }}$ (as labeled) everywhere in the column. After convergence, particles are vertically redistributed by sedimentation and mixing (standard scenario).

is added, identical to the first one except for mixing values enhanced by a factor of 2 . As for the last scenario, the enhancement factor is 5 . These mixing values were inspired by several works [Michelangeli et al., 1993; Colaprete et al., 1999]; indeed, they are recurrent in many studies. Martian atmospheric dynamics is assumed to feature strong updrafts, especially in the tropical band, where the Hadley circulation should be prevalent. Describing the three-dimensional subtleties of dynamics is a task beyond the capabilities of a 1-D model. For the current study we expect that our crude dynamics representation remains at a satisfactory level of sophistication.

[34] As alluded to earlier, our interest centers on the continuous portion of the J86 profile. The protocol of modeling was presented in section 3. For each dust model tested, we compare the resulting reflectance profile to the profile of J86. A major advantage of our method stems from its ability to place physical constraints on the effective

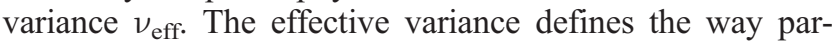
ticles are distributed among radii smaller and larger than the effective radius. $\nu_{\text {eff }}$ exerts a large influence on the way dust particles are vertically distributed, as depicted by Figure 7 (where the size at a given height is represented by $r_{\text {eff }}$ ).

[35] We previously discussed the importance of choosing a consistent size function type for dust aerosols, concluding that the lognormal form is the most appropriate and thus would be the one to adopt for the present study. A ques- tionable point concerns the fact of systematically assuming monomodal functions when extracting dust models from observations. We emphasize this topic later in this paper.

\subsubsection{Selected monomodal distribution}

[36] Figure 8 displays the results of the selected dust model ensuing from this analysis (hereinafter referred to as DM 1). This model is a narrow distribution of submicron particles $\left(r_{\text {eff }}=0.2 \mu \mathrm{m}, \nu_{\text {eff }}=0.03\right)$, for which best results were obtained for the intermediate mixing scenario. Owing to their low settling velocity, such small particles are easily maintained high in the atmosphere. As shown by Figure 8a, particle size is constant throughout the column. The morphology of the color ratio profile is indeed solely governed by the preferred extinction of blue. Scattered light has an initial color ratio value of 1.3 at $z=50 \mathrm{~km}$ as imposed by the ratio of the quantity $Q_{\text {ext }} \omega P(\theta)$. As it penetrates through the layer of these submicron particles, light becomes less "blue" owing to the depletion from the bluer part of its spectrum. These best fit values of effective radius and width are extremely sensitive to the variation of one of these two parameters. For instance, the effect of changing $\nu_{\text {eff }}$ to 0.1 while having the same $r_{\text {eff }}$ results in an offset of $-15 \%$ for
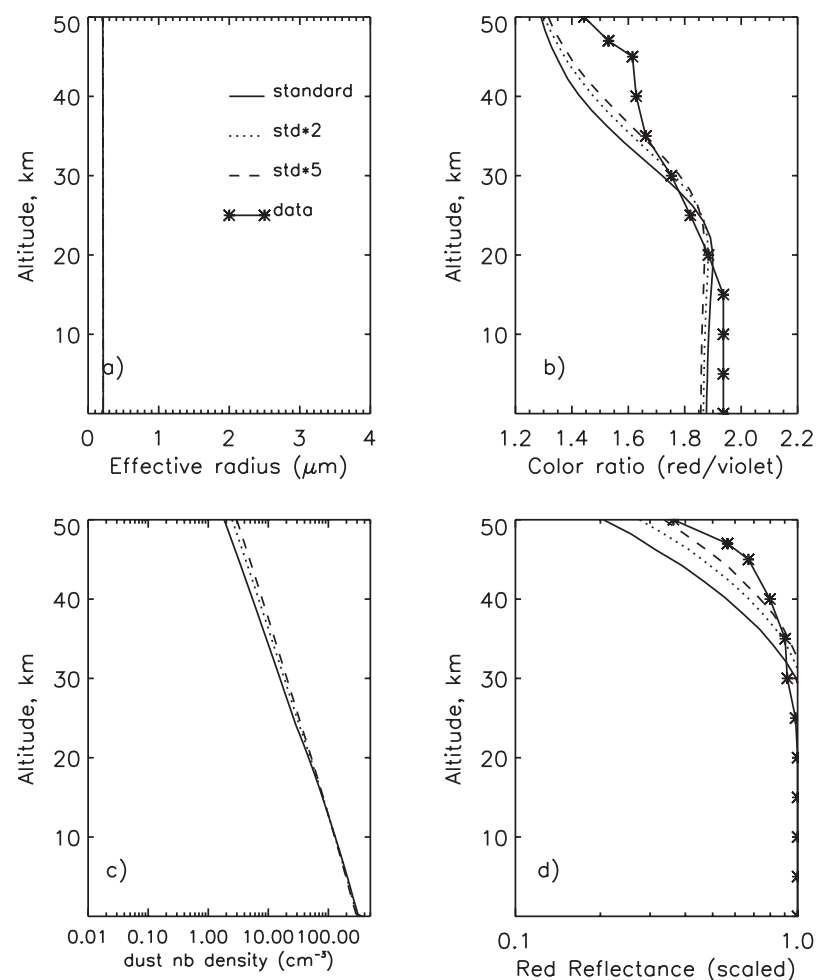

Figure 8. Results yielded by the preferred dust model (DM 1) defined by $r_{\text {eff }}=0.2 \mu \mathrm{m}$ and $\nu_{\text {eff }}=0.03$, in the case of simplified microphysical computations (no cloud scheme included). The three mixing scenarios used in this study are illustrated by different styles of curve (as labeled). Limb data points are reported in Figures $8 \mathrm{~b}$ and $8 \mathrm{~d}$. (a) Vertical profile of $r_{\text {eff. }}$ (b) Red to violet reflectance ratio versus height. (c) Plot of the dust particle number density as a function of height. (d) Reflectance profile for the red wavelength, where values are scaled by the one found near the ground, allowing a more convenient comparison with mata. 
the whole color ratio profile. In the same way, setting $r_{\text {eff }}$ at 0.1 while not changing $\nu_{\text {eff }}$ leads to an offset of $-50 \%$. On the other hand, these best fitting values are much more robust to changing the mixing scenario. It is somewhat suspicious to find such a lack of robustness and thus have a narrow range of fitting values. This latter point can be explained by the abrupt increase in the $Q_{\text {ext }}$ red/violet ratio within this particle size range (see Figure 4).

[37] However, according to J86, a gradual decrease of the color ratio from 2 to 1.5 between the ground and the top of the atmosphere is rather typical in the multiplecolor images of the Martian limb. Such a color behavior was also reported by Anderson and Leovy [1978]. Although not specified by these authors, this aspect of the Martian limb hazes is apparently independent of the location of sounding and moreover of the observation angle. Whereas the $Q_{\text {ext }} \omega P(\theta)$ ratio is also a function of the scattering angle $\theta$, observations of an unchanging color ratio pattern would provide strong evidences of such submicron particles.

\subsubsection{The "classical" dust model}

[38] Our results are in keeping with the Emission Phase Function sequences from Viking infrared thermal mapper (IRTM) observations derived by Clancy and Lee [1991]. Specifically, these authors reported dust effective radii 5-10 times smaller than previous analyses. As argued by Pollack et al. [1995], the range of scattering angles $\left(>60^{\circ}\right)$ employed for the Clancy and Lee retrieval is highly sensitive to particle shape. This comment is also true for our study of the Viking limb images. Even if current developments of optics allow the treatment of irregular particle shapes, no precise shape can be attributed to Martian aerosols. In addition, the semiempirical theory of Pollack and Cuzzi [1980], which offers a convenient design for simultaneous observations over a wide range of angles, requires intrinsic parameter adjustments incompatible with vertical profiling of particle size.

[39] However, a submicron dust issue contrasts strongly with the most widely accepted dust models to date [Tomasko et al., 1999; Pollack et al., 1995; Clancy et al., 1995; Korablev et al., 1993], the effective radius of which systematically exceeds $1 \mu \mathrm{m}$. Figure 9 presents simulation results while using the dust model inferred by Pollack et al. [1995] (referred to as DM 2). It is typical of the kind of fit obtained by dust models having effective size in the micron range $\left(\mathrm{r}_{\text {eff }}>1 \mu \mathrm{m}\right)$. Both modeled haze and color ratio profile do not match data. Owing to a larger average size relative to our selected model (DM 1), populations of particles centered on $\sim 1 \mu \mathrm{m}$ are confined to lower heights, lacking the cross-section area to fit the intensity profile at higher altitude. When applying enhanced eddy-mixing coefficients to counteract dust settling, a sufficient particle amount is available to enhance scattering at the desired elevations. On the other hand, this case approaches a uniformly mixed dust case where particle size is almost invariant with height. Such dust particles do not create contrasted extinction in the visible range (red to violet ratio of $Q_{\text {ext }} \sim 1$ ). As their color ratio profile follows the one of particle size, it largely deviates from observations. In short, attempts to simultaneously fit the red and blue channels of the Viking Orbiter Camera led to mutually
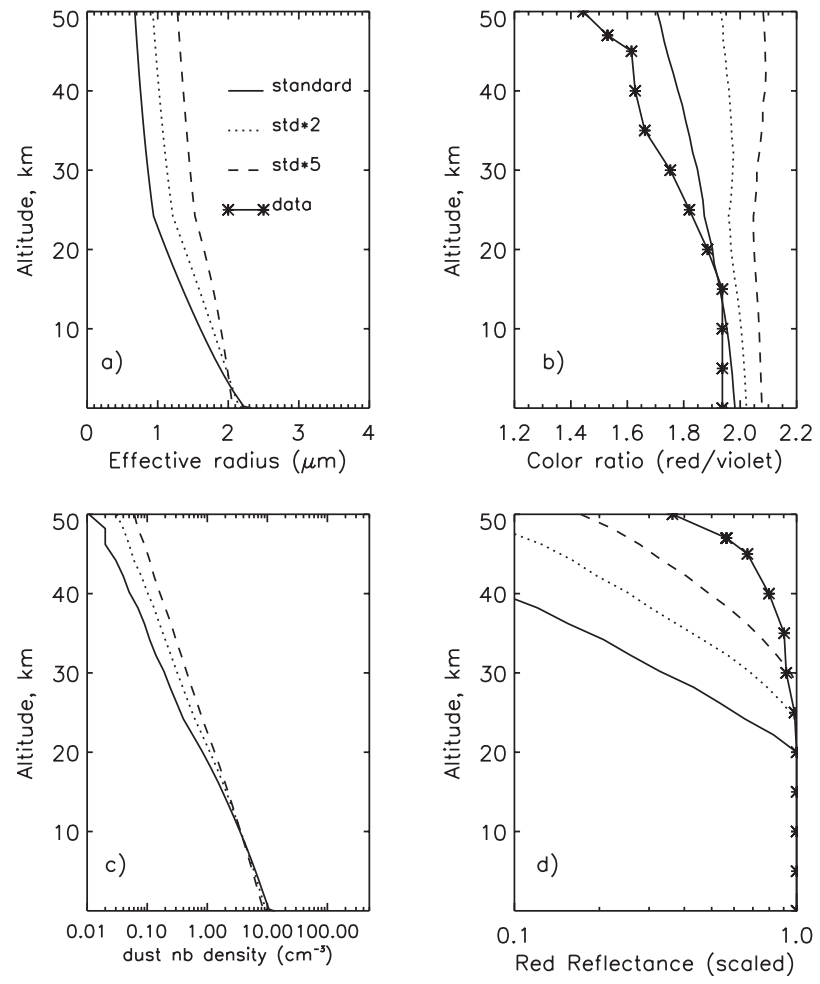

Figure 9. Same as Figure 8 but for the dust model (DM 2) inferred by Pollack et al. [1995] $\left(r_{\text {eff }}=1.8 \mu \mathrm{m}\right.$ and $\nu_{\text {eff }}=$ $0.5)$.

rejecting constraints. Although microphysically unstable, we tentatively assessed the behavior of the gamma-modified distribution of Clancy et al. [1995]. Such a model induces a larger population of submicron particles and thus is expected to give a better fit than DM 2 does. Subsequent results (not shown), though slightly better, are much poorer than those of DM 1, leading to a similar statement as for the case of DM 2.

\subsubsection{The bimodal hypothesis}

[40] Our selected monomodal model is not necessarily incompatible with the classical picture of Martian dust. We must propose a dust model which both explains the limb observations and is, however, consistent with previous

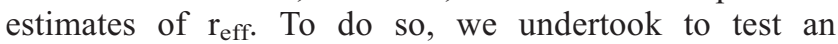
alternative case consisting of a bimodal size distribution for Martian dust. Adding three parameters (effective radius and width of the additional peak and relative population of one mode to the other) appears to prevent accurate extraction of the dust model when considering the amount of unknowns of the problem. The preliminary study helped us to formulate a reasonable guess of a relevant mode combination. Thus we formed our bimodal distribution (DM 3) by setting the main mode to the model of Pollack et al. [1995] (DM 2) and by adjusting an additional mode we supposed to be made of much smaller particles. It turns out that the best additional mode is identical to DM 1, while the population ratio of small to large particles is around 25 (Figure 13). Whereas the finer, easily lifted particles induce good structure of the color ratio as well as consistent intensity profiles above $20 \mathrm{~km}$, large particles 

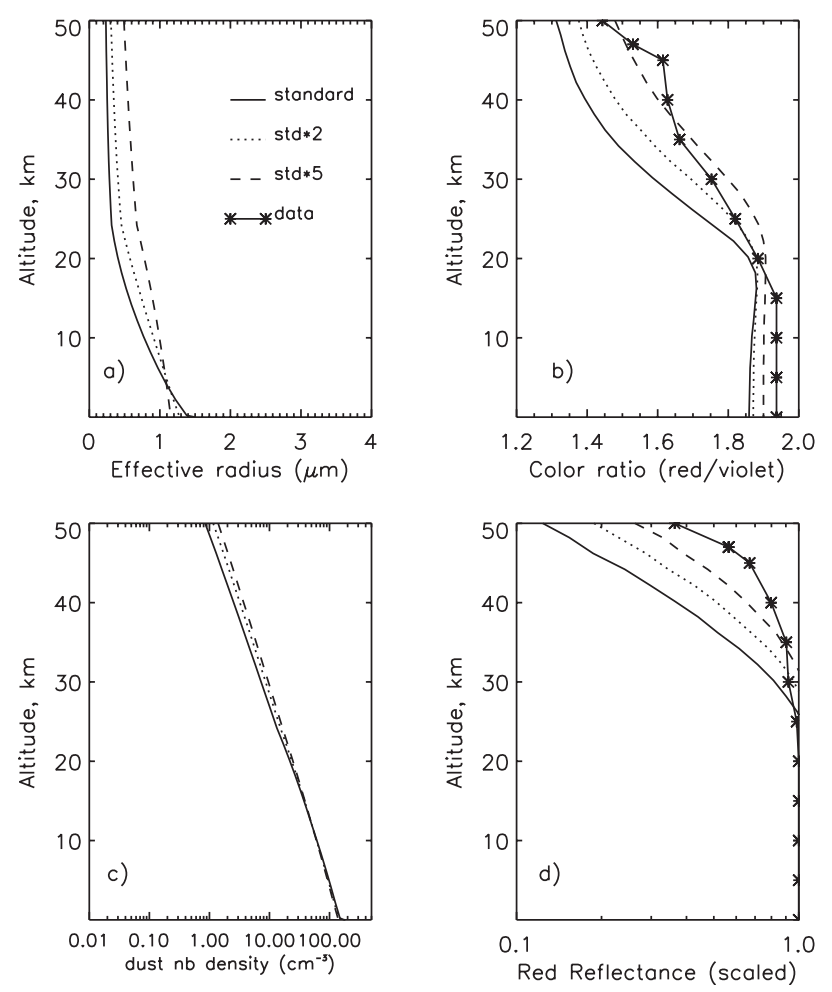

Figure 10. Same as Figure 8 but for the bimodal model (DM 3) selected by our analysis.

enhance somewhat the color ratio below this height (Figure 10).

\subsection{Simulations With Water-Ice Cloud}

[41] Considering a haze obtained while simulating waterice cloud formation provides additional constraints for the selection of a dust model. The preliminary assessment detailed in the previous section raised crucial requirements from the J86 multiple-color observations. The following analysis complements the previous one, which focused solely on the dusty portion of the J86 haze. The present part highlights the constraints yielded by the observed condensate layer (see Figure 3).

[42] The current knowledge about Martian water-ice clouds assumes that ice particles form onto airborne dust nuclei. Thus complex interactions exist between these two types of aerosols. Moreover, the formation and the evolution of a condensate cloud depend on the initial amount and on the supply of dust nuclei to the cloud level. As color ratios were also observed at the cloud elevation, we have precise constraints for the cloud pattern. For the present analysis, it helps us to indirectly verify the choice of our preferred dust model (i.e., the bimodal one) through the subsequent modeled cloud. Again with the observed color ratio and intensity profile, we can determine whether modeled profiles associated with any dust model are able to give a satisfactory fit.

[43] Using the EMCD database to get the likely temperature profile for the location and time of interest, we find a cloud forming at a much lower height than was observed by J86. Therefore an offset of $60^{\circ}$ in time was taken to obtain the desired water-ice cloud elevation. We assume an integrated water vapor content of 10 precipitable micrometers (pr $\mu \mathrm{m})$, similar to MAWD data at a period $\left(L_{s}=176^{\circ}\right)$ preceding dust storm seasons during which the MAWD detector was probably biased by shielding of dust [Smith, 2002].

[44] In order to emphasize the sensitivity of condensate cloud properties to varying dust models, we employed three different samples, that is, DM 1, DM 2, and DM 3 (our preferred model). The presence of a cloud is likely to affect the photometric analysis of the haze below it. A condensate cloud may produce significant spectral contrast and/or significant extinction before incident solar flux penetrates deeper in the atmosphere. This effect is strongly dependent on cloud opacity and cloud particle size. Consequently, it may more or less modify the interpretation of the portion of the profile assumed to be the result of scattering by dust. Fortunately, the J86 profile (Figure 3) appears to be mainly continuous while capped by a small detached layer at around $50 \mathrm{~km}$, the likely signature of a thin condensate
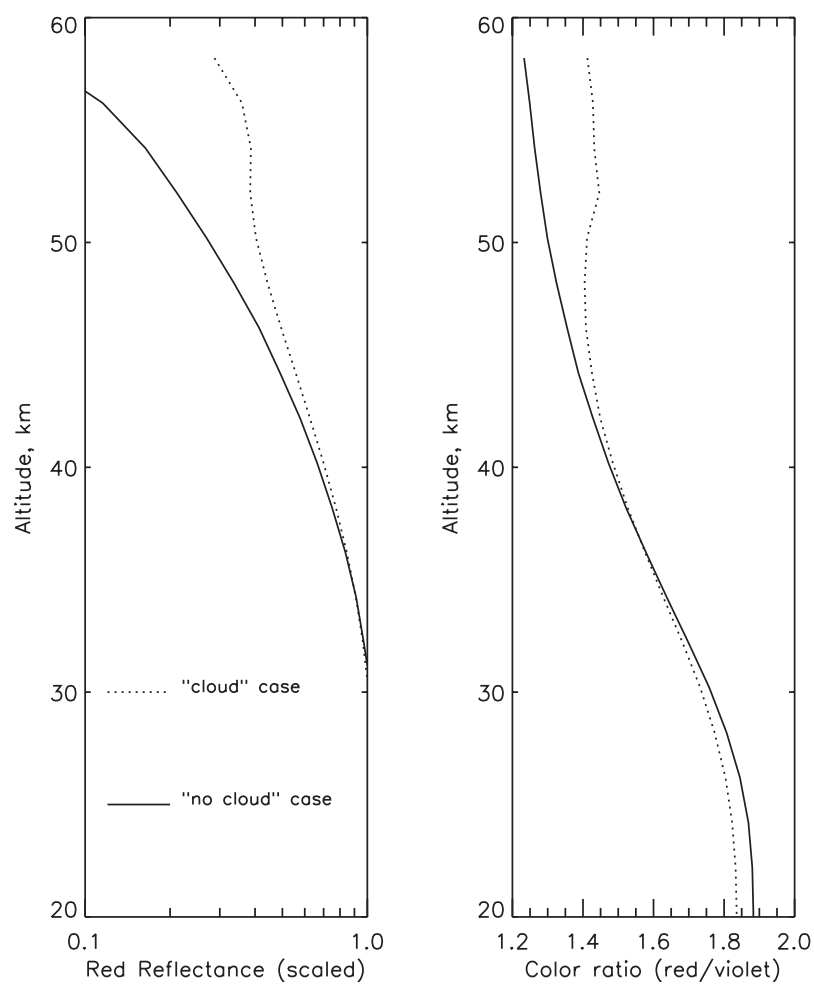

Figure 11. Comparison between photometric simulations based on the simplified microphysical scheme, the "no cloud case," and on the complete microphysical simulation, the "cloud case," including mixing and sedimentation but also nucleation and condensational growth of ice particles. Results for Dust Model 1 (DM 1) were taken for illustration. Ice particles allow larger geometric cross-section area and thus enhance light scattering (left panel) while changing color ratio (right panel). For the full microphysical scheme the subsequent presence of a condensate cloud at $50 \mathrm{~km}$ creates photometric deviations relative to the simplified scheme above $40 \mathrm{~km}$. Differences between the two cases become significant for elevations $>45 \mathrm{~km}$. 
Table 1. Modeled Cloud Characteristics ${ }^{\mathrm{a}}$

\begin{tabular}{cccccc}
\hline Dust Model Type & $r_{\text {eff }}^{\text {ice }}, \mu \mathrm{m}$ & $\nu_{\mathrm{eff}}^{\text {ice }}$ & $n_{\text {part }}, \mathrm{cm}^{-3}$ & $\begin{array}{c}\text { Cloud Opacity at } \\
\lambda=0.6 \mu \mathrm{m}\end{array}$ & Color Ratio \\
\hline DM 1 & 1.25 & 0.08 & 1.0 & 0.023 & 1.42 \\
DM 2 & 4.1 & 0.27 & 0.01 & 0.0023 & 1.67 \\
DM 3 & 1.8 & 0.05 & 0.6 & 0.012 & 1.52 \\
\hline
\end{tabular}

${ }^{\mathrm{a}}$ All values are given for $z=55 \mathrm{~km}$ except for the opacity (total cloud extinction).

cloud. To first order, neglecting cloud constraints did not mislead the photometric analysis made in the previous section. In Figure 11 we show a comparison of results for DM 1 obtained in cases of "cloud" and "no cloud" modeling. DM 1 was chosen since it yielded the largest condensate cloud opacity and the smallest ice particles of all chosen samples, thus generating the largest extinction and spectral contrast. It shall be considered as an optimum case for analyzing a condensate cloud impact on the underlying scattered light profile. Departure of "cloud" from the "no cloud" case is most significant at the cloud elevation. Surrounding elevations are not affected photometrically by the cloud presence unless within a range of $5 \mathrm{~km}$, specifically, between 45 and $60 \mathrm{~km}$.

[45] In all the cases of simulation, diurnal variations of temperature did not allow cloud opacity to fluctuate by more than a factor of 2 (not shown). All modeled clouds remain present throughout the day, exhibiting a peak of opacity at 0600 hours. At the local time of observations ( $~ 1000$ hours), the morning increase of temperature forces saturation to drop to a lower ratio but still to remain larger than unity. As argued by Chassefière et al. [1992], a steady state model is therefore appropriate to study such clouds formed at high altitudes.

[46] Table 1 summarizes several cloud characteristics for the three cases of simulation. Simulations with DM 2 exhibit the largest differences from the J86 data (Figure 12). The subsequent modeled cloud does not lead to a consistent reflectance curve, namely, 10 times less bright than the one yielded by data. Similarly, the modeled color ratio profile exhibits much greater values than the data, suggesting that modeled ice particles are too large. DM 2 results contrast with DM 1 and DM 3, which compare better with the cloud data, suggesting more realistic condensate cloud properties. Thus particles of the Viking cloud likely have an effective radius lying between 1.3 and $1.8 \mu \mathrm{m}$ at $55 \mathrm{~km}$, while the cloud opacity in the visible can be approximated by a value of 0.02. A slight difference between DM 1/DM 3 and data is observed, however, at the top of the color ratio profile. DM 1 and DM 3 have their ratio constant above $55 \mathrm{~km}$, whereas observations show an inversion above this height, suggesting a decrease of the water-ice particle size. It is likely the consequence of an inappropriate mixing strength in this part of the atmosphere. Since it has no consequence for the remainder of the analysis, we do not give further attention to this point.

[47] DM 1 and DM 3 results provide strong evidence that a large amount of dust nuclei is necessary for water vapor to condense, in order that modeled clouds agree with the detached layer of the observation. For an equivalent volume of water ice, the associated cross-section area of condensate particles is inversely proportional to their mean radius. The latter can be roughly viewed as inversely proportional to the cubic root of the dust nucleus number density. Again, DM 2 suffers from the problem of dust confinement within lower layers. In comparison, dust models of finer particles require a more important amount of particles in order to obtain the same visible opacity. Since their size favors their lifting toward a higher altitude, where condensation occurs, such dust models supply larger amounts of nuclei for the nucleation process. This provides a straightforward explanation to the fact that larger condensate cloud opacities are found for DM 1 and DM 3 relative to DM 2.

[48] The results of this section confirm the conclusion we drew in the previous section although in this section only the vertical structure analysis of the dust haze was used. Extended microphysical modeling requires a small dust particle population in the higher region of the atmosphere in order to form a condensate cloud agreeing with data. Our results can be compared with those of the Phobos clouds studied by Chassefière et al. [1992]. These
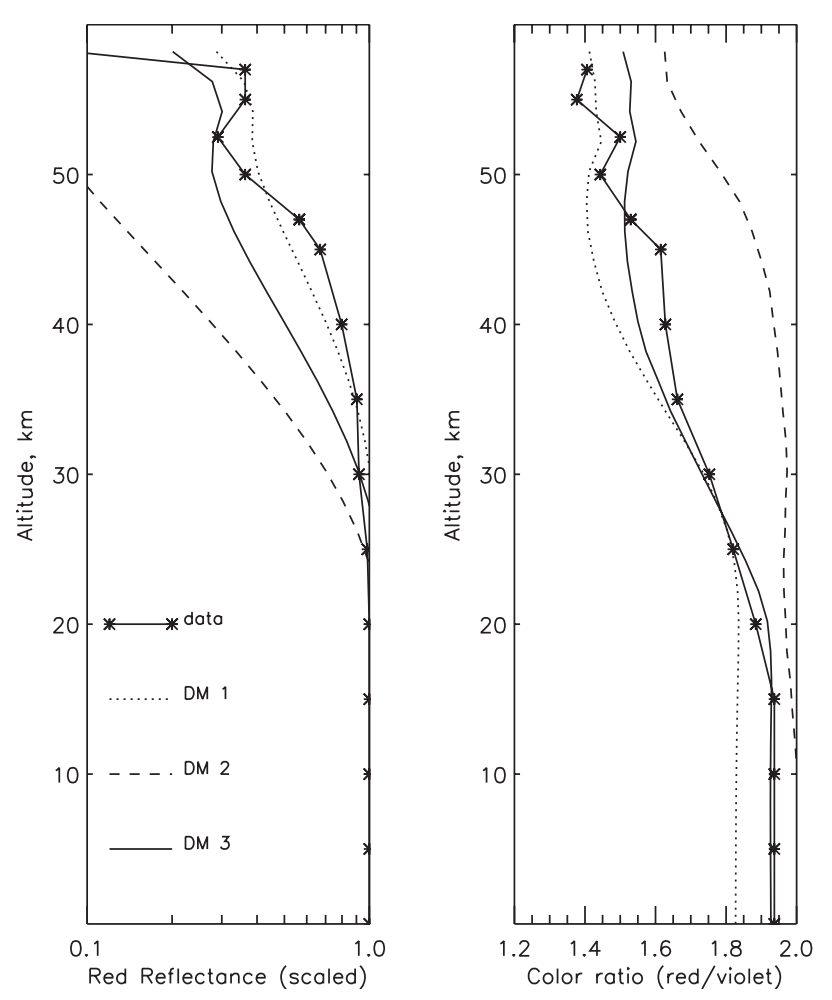

Figure 12. Summary of the cloud simulations. The same presentation is used as in Figure 11. Results for DM 1, 2, and 3 are compared to limb data. 
authors derived similar values of cloud particle number density $\left(\sim 1 \mathrm{~cm}^{-3}\right)$ from solar occultation at elevations higher than $40 \mathrm{~km}$. This also implies that a large amount of dust nuclei was present to allow the formation of these clouds and thus confirms the present analysis of the cloud of J86. On the other hand, particles had smaller sizes, typically below $1 \mu \mathrm{m}$. However, these facts added together provide additional arguments to support our hypothesis that the Martian dust distribution is most likely to be bimodal.

\section{Discussion}

[49] A new perspective has been given to the Viking limb images of the Martian limb. Despite the limited set of images investigated in this study (only one pair), several implications were drawn from the simultaneous analysis of the same limb using two visible wavelengths (red and blue). Having many parameters to deal with complicates the task of limb data derivation. From a technical point of view, we are now able to give more accurate interpretations of the observed profile of the color ratio. A color ratio difference between the ground haze and the higher altitude is commonly attributed to the presence of "bluer" condensate particles. We demonstrated that dust particles play a key role in the color ratio pattern exhibited by the observations of J86. Our study favored some elementary physical constraints in order to satisfy observational requirements. We are drawn to the conclusion that small dust particles dominate the upper atmospheric region $(>15 \mathrm{~km})$ seen by Viking. Such a type of dust $\left(r_{\mathrm{eff}}>\right.$ $0.2 \mu \mathrm{m}$ ) is necessary not only to match the observed color ratio behavior in the dusty portion but to obtain consistent condensate cloud properties too. In this way, we estimate the particle effective radius of the cloud seen by Viking to be around $1.5 \mu \mathrm{m}$ with an effective width of 0.03 . Cloud opacity should slightly exceed 0.02 in the visible range. The values obtained here are in remarkable agreement with the most recent observations of water ice clouds of Pearl et al. [2001]. Spectral signatures of clouds are found to be surprisingly invariant with seasons, implying unchanged particle size $\left(r_{\text {eff }} \sim 2 \mu \mathrm{m}\right)$.

[50] However, while accounting for other observation retrievals [Korablev et al., 1993; Pollack et al., 1995; Tomasko et al., 1999], we postulated that Martian dust distribution was potentially bimodal. Indeed, the simultaneous presence of two peaks in the size spectrum provided significant improvements to modeled reflectance profiles. This hypothesis has already been formulated in previous studies [Chassefière et al., 1992; Markiewicz et al., 1999]. Markiewicz et al. [1999] reported an increasing effective dust radius with wavelength strongly suggesting an unaccounted contribution of Rayleigh scatterers, namely, small particles. Wolff et al. [2001] presented recent observations from the Emission Phase Function of the Thermal Emission Spectrometer on board Mars Global Surveyor. Their results give similar values for the size parameter of dust as the common insight does. They also detected substantial seasonal and spatial changes of dust single scattering properties. Specifically, they found an increase of the visible to infrared ratio, implying a significant drop of particle effective size.

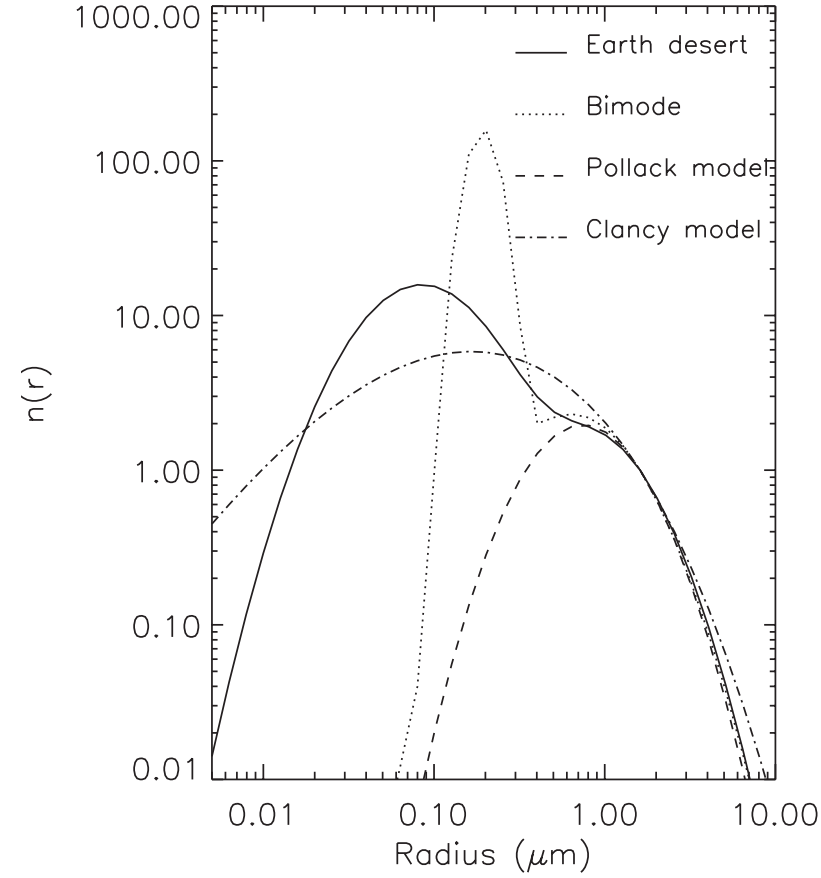

Figure 13. A variety of dust size distributions derived from observations of the Martian atmosphere, including the one resulting from this work. In order to make a more convenient comparison, each distribution is normalized to the value found at a radius of $1.6 \mu \mathrm{m}$. A dust distribution of the Saharan desert is also reported to show the strong similarities existing between the two species of aerosols in terms of granulometry. One can notice the difference in width of the small particle mode between our bimodal distribution and the terrestrial sample. It can be shown, however, that the strength of the coagulation process on Mars prevents the stability of such a broad submicron mode. On Earth this effect is found to exert a lower influence.

[51] On the basis of our own analysis, together with the studies mentioned above, we feel that the "classical" picture of Martian dust should be revised. It makes sense to address the issue of the dust size function in this case. Our investigations of a bimodal form led us to conclude that it potentially had the best scattering properties to match series of observations. Measurements performed in arid regions of Africa are of great interest to investigate soilderived aerosol behavior within environments resembling those of the Martian surface [d'Almeida, 1987]. It is interesting to find that Earth desert aerosols exhibit striking similarities to Martian species. A first point concerns the classification of weather conditions made by this author; fair-weather, sandstorm episodes are also found to occur in the Martian atmosphere. All size distributions derived by d'Almeida [1987] yield strong absorption peaks at $10 \mu \mathrm{m}$, clogging the atmospheric window as does Martian airborne dust in this spectral region. A siliceous clay component is assumed to induce such radiative properties in both cases. More striking is the visible to infrared ratio of $\sim 2$ one can readily infer from the work of d'Almeida [1987]. This ratio is found to decrease somewhat during sandstorm episodes. Its Martian counterpart has been widely documented, and a 
canonical value for this ratio has been adopted, namely, 2.5 [Martin, 1986; Toigo and Richardson, 2000]. Toigo and Richardson [2000] specifically focused on the ratio changes with season, arguing that a previous underestimate of waterice cloud contribution explained these changes.

[52] Obviously, sandblasting is the common source process for both Martian and Earth desert soil-derived aerosols. Whereas it is not clear how this process could generate simultaneously more than one size mode, size functions of Saharan levitated dust exhibit separate peaks. Figure 13 shows a comparison between a sand size spectrum derived by d'Almeida [1987] and several models of Martian dust, including the one we extracted in our analysis of the Viking limb images. It strongly supports the bimodal hypothesis for Martian dust. The terrestrial coarse mode (right peak of the spetrum) is identical to those inferred by Martian atmosphere soundings. Another insight has been given by Gomes et al. [1990] through their experiments in the Saharan desert using a cascade impactor. These experiments allowed the derivation of airborne dust size functions. Function shapes similar to those of d'Almeida [1987] were obtained, namely, multimodal. These authors inquired into the origins of the submicron peak. By means of scanning electron micrographs, Gomes et al. [1990] were able to show that the mode of submicron particles had the same mineralogical composition as the coarse mode. Furthermore, given the dramatic increase of the submicron particle population during sandstorm episodes, the bimodal form of the size function became more apparent. Thus wind stress increases correspond to larger injections of submicron dusts into the atmosphere. Therefore they had irrefutable evidence that these submicron particles were the result of a sandblasting process. Multimodal distributions for soil-derived aerosols are a potential consequence of lifting by winds and thus are a likely alternative for the Martian dust distribution.

[53] Acknowledgments. We wish to thank Francois Forget and Wojtek Markiewicz for useful discussions. We also benefited from the very constructive review of M. D. Smith, the suggestions and comments of which deeply improved this work.

\section{References}

Anderson, D. W., M. J. Schwarz, and A. R. Tice, Water vapor adsorption by sodium montmorillonite at $-5^{\circ} \mathrm{C}$, Icarus, 34, 638-644, 1978.

Anderson, E., and C. Leovy, Mariner 9 television limb observations of dust and ice hazes on Mars, J. Atmos. Sci., 35, 723-734, 1978.

Bertaux, J. L., and F. Montmessin, Isotopic fractionation through water vapor condensation: The Deuteropause, a cold trap for Deuterium in the atmosphere of Mars, J. Geophys. Res., 106, 32,863-32,878, 2001.

Cabane, M., E. Chassefière, and G. Israel, Formation and growth of photochemical aerosols in Titan's atmosphere, Icarus, 96, 176-189, 1992.

Chassefière, E., J. E. Blamont, V. A. Krasnopolsky, O. I. Korablev, S. K

Atreya, and R. A. West, Vertical structure and size distributions of Martian aerosols from solar occultation measurements, Icarus, 97, 46-69, 1992.

Chassefière, E., P. Drossart, and O. Korablev, Post-Phobos model for the altitude and size distribution of dust in the low Martian atmosphere, J. Geophys. Res., 100, 5525-5539, 1995.

Clancy, R. T., and S. W. Lee, A new look of emission-phase-function sequences from global Viking IRTM observations, Icarus, 93, 135$158,1991$.

Clancy, R. T., S. W. Lee, G. R. Gladstone, W. W. McMillan, and T. Rousch, A new model for Mars atmospheric dust based upon analysis of ultraviolet through infrared observations from Mariner 9, Viking, and Phobos, J. Geophys. Res., 100, 5251-5263, 1995.

Colaprete, A., and O. B. Toon, The radiative effects of Martian water ice clouds on the local atmospheric temperature profile, Icarus, 145, 524$532,2000$.
Colaprete, A., O. B. Toon, and J. A. Magalhães, Cloud formation under Mars Pathfinder conditions, J. Geophys. Res., 104, 9043-9053, 1999.

Colburn, D. S., J. B. Pollack, and R. M. Haberle, Diurnal variations in optical depth at Mars, Icarus, 79, 159-189, 1989.

d'Almeida, G. A., On the variability of desert aerosols radiative characteristics, J. Geophys. Res., 92, 3017-3026, 1987.

Epstein, B., The mathematical description of certain breakage mechanisms leading to the logarithmico-normal distribution, J. Franklin Inst., 244, 471-477, 1947.

Fenton, L. K., J. C. Pearl, and T. Z. Martin, Mapping Mariner 9 dust opacities, Icarus, 130, 115-125, 1997.

Forget, F., F. Hourdin, R. Fournier, C. Hourdin, O. Talagrand, M. Collins, S. R. Lewis, P. L. Read, and J. P. Huot, Improved general circulation models of the Martian atmosphere from the surface to above $80 \mathrm{~km}$, J. Geophys. Res., 104, 24,155-24,176, 1999.

Friedlander, S. K., Smoke, Dust and Haze, Wiley-Interscience, New York, 1977.

Fuchs, N., The Mechanics of Aerosols, Pergamon, New York, 1964.

Gierasch, P. J., and R. M. Goody, The effect of dust on the temperature of the Martian atmosphere, J. Atmos. Sci., 29, 400-402, 1972.

Gomes, L., G. Bergametti, G. G. Coudé-Gaussen, and P. Rognon, Submicron desert dusts: A sandblasting process, J. Geophys. Res., 95(D9), 13,927-13,936, 1990.

Gooding, J. L., Martian dust as condensation nuclei: A preliminary assessment of mineralogical factors, Icarus, 66, 55-74, 1986.

Hansen, J. E., and L. D. Travis, Light scattering in planetary atmospheres, Space Sci. Rev., 16, 527-560, 1974.

Jaquin, F., P. Gierasch, and R. Kahn, The vertical structure of limb hazes in the Martian atmosphere, Icarus, 68, 442-461, 1986.

Kahn, R., Ice haze, snow, and the Mars water cycle, J. Geophys. Res., 95, 14,677-14,693, 1990.

Keese, R. G., Nucleation and particle formation in the upper atmosphere, J. Geophys. Res., 94, 14,683-14,692, 1989.

Korablev, O. I., V. A. Krasnopolsky, A. Rodin, and E. Chassefiere, Vertical structure of Martian dust measured by solar infrared occultations from the Phobos spacecraft, Icarus, 102, 76-87, 1993.

Kuroda, T., Rate determining process of growth of ice crystals from the vapour phase, part I, Theoretical consideration, J. Meteorol. Soc. Jpn., 62, 552-561, 1984.

Lewis, S. R., M. Collins, P. L. Read, F. Forget, F. Hourdin, R. Fournier, C. Hourdin, O. Talagrand, and J. P. Huot, A climate database for Mars, J. Geophys. Res., 104, 24,177-24,194, 1999.

MacKenzie, A. R., and P. H. Haynes, The influence of surface kinetics on the growth of stratospheric ice crystals, J. Geophys. Res., 97, 80578064, 1992.

Markiewicz, W. J., R. M. Sablotny, H. U. Keller, and N. Thomas, Optical properties of the Martian aerosols as derived from Imager for Mars Pathfinder midday sky brightness data, J. Geophys. Res., 104, 9009-9017, 1999.

Martin, T. Z., Thermal infrared opacity of the Mars atmosphere, Icarus, 66, 2-21, 1986

Michelangeli, D. V., O. B. Toon, R. B. Haberle, and J. B. Pollack, Numerical simulations of the formation and evolution of water ice clouds in the Martian atmosphere, Icarus, 100, 261-285, 1993.

Mooney, R. W., A. G. Keenan, and L. A. Wood, Adsorption of water vapor by montmorillonite, I, Heat of desorption and application of BET theory, J. Am. Chem. Soc., 74, 1367-1371, 1952.

Ockert-Bell, M. E., J. F. Bell III, J. P. Pollack, C. P. McKay, and F. Forget, Absorption and scattering properties of the Martian dust in the solar wavelength, J. Geophys. Res., 102, 9039-9050, 1997.

Pearl, J. C., M. D. Smith, B. J. Conrath, J. L. Bandfield, and P. R. Christensen, Observations of Martian ice clouds by the Mars Global Surveyor Thermal Emission Spectrometer: The first Martian year, J. Geophys. Res., 106, 12,325-12,338, 2001.

Pollack, J. B., and J. N. Cuzzi, Scattering by nonspherical particles of size comparable to a wavelength: A new semi-empirical theory and its application to tropospheric aerosols, J. Atmos. Sci., 37, 868-881, 1980.

Pollack, J. B., M. E. Ockert-Bell, and M. K. Shepard, Viking Lander analysis of Martian atmospheric dust, J. Geophys. Res., 100, 5235$5250,1995$.

Pruppacher, H. R., and J. D. Klett, Microphysics of Clouds and Precipitation, D. Reidel, Norwell, Mass., 1978.

Rages, K., and J. B. Pollack, Vertical distribution of scattering hazes in Titan's upper atmosphere, Icarus, 55, 50-62, 1983.

Rannou, P., M. Cabane, R. Botet, and E. Chassefière, A new interpretation of scattered light measurements at Titan's limb, J. Geophys. Res., 102, 10,997-11,013, 1997.

Rodin, A. V., O. I. Korablev, and V. I. Moroz, Vertical distribution of water in the near-equatorial troposphere of Mars: Water vapor and clouds, Icarus, 125, 212-229, 1997. 
Seki, J., and H. Hasegawa, The heterogeneous condensation of interstellar ice-grains, Astrophys. Space Sci., 94, 177-189, 1983.

Smith, M. D., The annual cycle of water vapor on Mars as observed by the Thermal Emission Spectrometer, J. Geophys. Res., 107, 10.1029/ 2001JE001522, in press, 2002.

Smith, M. D., J. C. Pearl, B. J. Conrath, and P. R. Christensen, Thermal Emission Spectrometer results: Mars atmospheric thermal structure and aerosol distribution, J. Geophys. Res., 106, 23,929-23,945, 2001.

Toigo, D. A., and M. I. Richardson, Seasonal variation of aerosols in the Martian atmosphere, J. Geophys. Res., 105, 4109-4121, 2000.

Tomasko, M. G., L. R. Doose, M. Lemmon, P. H. Smith, and E. Wegryn, Properties of dust in the Martian atmosphere from the Imager on Mars Pathfinder, J. Geophys. Res., 104, 8987-9007, 1999.

Toon, O. B., J. B. Pollack, and C. Sagan, Physical properties of the particles composing the Martian dust storm of 1971-1972, Icarus, 30, 663-696, 1977.

Toon, O. B., R. P. Turco, D. Westphal, R. Malone, and M. S. Liu, A multidimensional model for aerosol: Description of computational analogs, J. Atmos. Sci., 45, 2123-2143, 1988.

Toon, O. B., R. P. Turco, J. Jordan, J. Goodman, and G. Ferry, Physical processes in polar stratospheric ice clouds, J. Geophys. Res., 94, 11,35911,380, 1989a.
Toon, O. B., C. P. McKay, and T. P. Ackerman, Rapid calculation of radiative heating rates and photodissociation rates in inhomogeneous multiple scattering atmospheres, J. Geophys. Res., 94, 16,287-16,301, 1989 b.

Warren, S. G., Optical constants of ice from the ultraviolet to the microwave, Appl. Opt., 23, 1206-1225, 1984.

Wolff, M. J., R. T. Clancy, K. M. Pitman, P. R. Christensen, and Whitney, Mars aerosol studies with the MGS TES emission phase function observations: Opacities, particle sizes, and ice cloud types, paper presented at DPS meeting 33, Am. Astron. Soc., New Orleans, La., 2001.

Zent, A. P., and R. C. Quinn, Measurements of $\mathrm{H}_{2} \mathrm{O}$ adsorption under Marslike conditions: Effects of adsorbent heterogeneity, J. Geophys. Res., 102, 9085-9095, 1997.

Zhang, M. H. G., J. G. Luhmann, A. J. Kliore, and J. Kim, A post-Pioneer Venus reassessment of the Martian dayside ionosphere as observed by radio occultation method, J. Geophys. Res., 95, 14,829-14,839, 1990.

M. Cabane, F. Montmessin, and P. Rannou, Service d'Aéronomie du CNRS, Université Pierre et Marie Curie, Tour 15, B 102, 4 Place Jussieu, 75252 Paris Cedex 05, France. (montmes@aero.jussieu.fr) 